\title{
Oncological Emergencies in Chest Medicine
}

\author{
Gamal Agmy* \\ Respiratory medicine, Assiut University, Egypt
}

*Corresponding author: Gamal Agmy, Respiratory medicine, Assiut University,

Egypt, Email: gamalagmy135@gmail.com

\section{Review Article \\ Volume 3 Issue 1}

Received Date: May 02, 2018

Published Date: June 06, 2018
Abbreviations: VTE: Venous Thrombo Embolism; TLS: Tumor Lysis Syndrome; SVC: Superior Vena Cava; SIADH: Syndrome of Inappropriate secretion of Anti Diuretic Hormone; MRI: Magnetic Resonance Imaging.

\section{Pulmonary Embolism}

Lung cancer is the most common malignancy coexisting in patients with venous thromboembolism (VTE) [1]. A large population-based, case-control study demonstrated that patients with hematologic malignancies had the highest risk of VTE, followed by patients with lung and gastrointestinal cancers [2].

Adenocarcinoma of the lung had a higher risk of thromboembolic events [3]. The effects of mucin production on procoagulatory factor activation may trigger thromboembolic events [4]. Blom, et al. [5] demonstrated that the risk of VTE in lung cancer patients increased 20-fold compared to the general population and that patients with adenocarcinoma have a higher risk of VTE than patients with squamous cell carcinoma.

\section{Tumor Lysis Syndrome}

Tumor lysis syndrome (TLS) occurs when there is rapid cell breakdown after chemotherapy most commonly for leukaemias and higher grade lymphomas with large tumor burdens that respond quickly to chemotherapy [6]. The main manifestations of tumor lysis syndrome are hyperkalemia, hyperphosphatemia, hypocalcemia, hyperuricemia, and azotemia. The metabolic changes are essentially caused by cell breakdown products. Massive cell death and nuclear products liberated nucleic acids and adenine and guanine are broken down to uric acid which precipitates as urate crystals and causes urate renal failure. Lactic acidosis can occur [7] to try to prevent tumourlysis syndrome, patients should be hydrated to achieve a high urine output and treated with allopurinol or rasburicase to decrease uric acid. Once tumourlysis is established patients may require dialysis [8].

\section{Massive Hemoptysis}

Etiology of hemoptysis in most malignant diseases includes severe neutropenia and / or thrombocytopenia due to cytotoxic drugs or the bone marrow infiltration. Coagulopathy due to decreased liver production of clotting factors secondary to extensive hepatic metastases is another important issue. Infections especially of fungal origin play an important role in creating hemoptysis in hematologic malignancies and bone marrow transplantation. In conclusion, the main causes of life threatening hemoptysis among cancer patients include haematological abnormalities, diffuse alveolar damage, coagulopathy, fungal, viral and bacterial infections, sepsis, radiation lung injury, and lung damage secondary to chemotherapy [9].

\section{Superior Vena Cava Obstruction}

Bronchogenic carcinoma is the main reason superior vena cava (SVC) thrombosis and the superior vena cava syndrome (SVCS) followed by lymphoma and mediastinal metastases then indwelling catheters for vascular access [10].

The treatment is tailored by the presentation of SVCS and the cell form of malignancy. Urgent radiotherapy is rarely needed preceding a diagnosis. Primary actions include head elevation, supplemental oxygen and restricted use of either intravenous fluids or diuretics. Steroids are mandatory in presence of respiratory or CNS manifestations.10 Radiotherapy is the main therapeutic modality with increased dose fractions (300 to $400 \mathrm{cGy}$ ) on the first 3 days followed by full course radiation at conventional dose fractions (180 to $200 \mathrm{cGy}$ ) and up to 3,000 to 5,000 cGy, depending on cancer cell type. 


\section{Open Access Journal of Pulmonary \& Respiratory Sciences}

Chemotherapy may be used in conjunction with radiotherapy for lymphomas, while, used unaided for small-cell lung cancer [10].

Bronchoscopic maneuvers including stents, thrombolytic therapy and angioplasty are growing. Streptokinase or urokinase may be useful if it is used within 7 days of the onset of symptoms. Successful resolution of thrombosis is improbable after 1 week [10].

\section{Cardiac Tamponade}

It is caused mainly by metastatic tumors especially those from lung, breast, lymphoma, leukemia, and melanoma. Uremia, cytotoxic drugs, or radiation injury to the pericardium may be an alternative causes [11]. The best diagnostic modality is the echocardiogram by detection of pericardial effusion with collapse of the right atrium and right ventricle duing diastole. Right heart catherization will reveal increase and equalization of pressures of the right atrium, the right ventricle, the pulmonary artery in diastole, and the pulmonary capillary wedge pressure. The right atrial pressure tracing and the clinical JVP will establish a prominent X descent [12].

The treatment modality of choice is pericardiocentesis for abrupt relief of symptoms. The aspirated fluid should be sent for laboratory chemical and cytologic examinations. Systemic chemotherapy, catheter drainage and sclerosis, radiation therapy, or surgical interventions are therapeutic approaches to prevent accumulation of fluid [11]. Pericardiectomy, pleuropericardial window, or subxiphoidpericardiotomy are the available surgical options. Patients should be started on the proper systemic therapy simultaneously if their performance status permits [12].

\section{Syndrome of Inappropriate Secretion of Antidiuretic Hormone (SIADH)}

Hyponatremia with inappropriately concentrated urine are the main manifestations of SIADH. The most common cause is small cell lung cancer accounting for $60 \%$ of all cases of SIADH. The differential diagnosis of SIADH includes side effects of cytotoxic chemotherapy agents, particularly cyclophosphamide and vincristine, and ectopic creation of atrial natriuretic factor [13].

Treatment comprises handling the primary tumor producing the antidiuretic hormone or atrial natriuretic factor beside fluid management, frequently fluid restriction or induced diuresis. Proper combination chemotherapy should be started, and brain metastases, if existing, should be managed with radiotherapy [14].

Fluid consumption should be restricted to less than one liter /day and less than $500 \mathrm{~mL} /$ day if the patient response is underprivileged. Resistant cases of hyponatremia or patients who can be managed as outpatients can be treated with 600 to $1,200 \mathrm{mg} / \mathrm{d}$ of demeclocycline (Declomycin) in distributed doses. Hypertonic saline 3\% is indicated in patients with coma or seizures by slow infusion at a rate sufficient to increase the serum sodium level by 0.5 to $1.0 \mathrm{mEq} / \mathrm{L} / \mathrm{h}$.Rapid infusion must be avoided (greater than $2 \mathrm{mEq} / \mathrm{L} / \mathrm{h}$ ) as it may be linked with central pontinemyelinolysis. Neutral saline 0.9 with IV furosemide may be effective [14].

\section{Hypercalcemia of Malignancy}

This condition most commonly is associated with multiple myeloma and cancers of the lung, breast, and kidney. Mechanisms that are thought to be important in the development of hypercalcemia of malignancy include bone-resorbing cytokines; parathyroid hormone-related peptide, secreted by the tumor that binds to parathyroid hormone receptors; tumor-mediated calcitriol production; and occasionally ectopic parathyroid hormone secretion [15].

The best therapeutic modality for hypercalcemia of malignancy is aggressive rehydration followed by diuresis with furosemide. Check of serum phosphorus is mandatory because hypophosphatemia is common and can deteriorate the condition [15]. Phosphorus should be substituted orally or via a nasogastric tube. Intravenous bisphosphonate therapy can hinder osteoclastic bone resorption [16]. The quality of life in patients with metastatic bone disease is markedly improved by use of Zoledronic acid and pamidronate by decreasing skeletal complications, bone pain, and the need for analgesics. Dialysis and glucocorticoid, calcitonin (Miacalcin), plicamycin (Mithracin), and gallium nitrate (Ganite) therapies are adjunctive therapeutic options [17].

\section{Epidural Spinal Cord Compression}

If treatment is delayed for only a few hours, a permanent neurologic impairment can occur. Epidural spinal cord compression is related to breast and lung cancers followed by renal and prostate, cancers. The common site affected is the thoracic spine, recorded in 70 $\%$ of patients with this condition. Early interventions in patients with epidural spinal cord compression improve outcomes. Almost $90 \%$ of patients who are ambulatory at 


\section{Open Access Journal of Pulmonary \& Respiratory Sciences}

the time of diagnosis do not miss this capability post treatment [18].

Suggests Epidural spinal cord compression must be suspected in any cancer patient with back pain. Pain that deteriorates when the patient is lying down or with percussion of vertebral bodies is specific for this condition. Incontinence and loss of sensory function are accompanied with permanent paraplegia. Plain radiograph is usually diagnostic in patients with solid tumors [19]. The imaging of choice is magnetic resonance imaging (MRI) Steroid therapy in the form of dexamethasone bolus (10 mg intravenously) followed by 4-mg doses every six hours should be started in the presence of neurological symptoms not awaiting diagnostic results. Radiation treatment (up to 3,000 Gy) or surgery is usually needed. Immediate radiation therapy is mandatory in asymptomatic patients, and patients with progressive symptoms in spite of radiation should be subjected for surgical intervention [20].

\section{Febrile Neutropenia}

Cancer treatment, particularly chemotherapy is usually complicated by febrile neutropenia. Febrile neutropenia accounting for 50 percent of deaths related to leukemia, lymphomas, and solid tumors. Bacterial infections are common in patients with febrile neutropenia, but fungal infections are progressively dominant. The predominant symptoms are fever with an absolute neutrophil count less than 500 per $\mathrm{mm} 3(0.5 \times 109$ per L) [21].

A prospective, multinational study of 1,139 patients with febrile neutropenia authorized a scoring system to categorize patients as high or low risk. When grampositive and gram-negative organisms are suspected, multi-drug regimens are applied. Linzolides or vancomycinae added when MRSA is suspected. Antifungals are indicated if there is no progress within the first three days of treatment. Antivirals, granulocyte transfusions, and colony-stimulating factors are not usually recommended [21].

\section{Hyper Viscosity Syndrome}

Hyper viscosity syndrome is more prevalent in patients with leukemia, multiple myeloma or Waldenström's macroglobulinemia. Increased levels of circulating serum immunoglobulin coat the cells, causing increased blood viscosity, slowing of blood, and hypo perfusion [22].

The main symptoms incorporate spontaneous bleeding, neurologic defects and vision changes. Sausage-like" hemorrhagic retinal veins are pathognomic. Hyper viscosity syndrome should be suspected when serum viscosity exceed $5 \mathrm{cP}$. Plasmapheresis is the treatment of choice; and it should be repeated in refractory episodes; followed by targeted chemotherapy [23].

\section{Acute Airway Obstruction}

Acute airway obstruction involves the upper airways and may be caused by malignant or non-malignant conditions. This term refers to a blockage at the level of the main stem bronchi or above. It may result from intraluminal tumor growth or from extrinsic compression of the airway. Tumors that obstruct the upper airway by direct extension are primary tumors of the head and neck and lung. Primary tumors of the lung are the most common cause of lower airway obstruction. Dyspnea is frequently the only early symptom of airway obstruction. If dyspnea occurs on exercise, the airway diameter is decreased to $8 \mathrm{~mm}$, but if dyspnea occurs at rest, the airway diameter is usually compression is the main cause of obstruction; stent placement is the preferred method of palliation [24].

\section{Massive Pleural Effusion}

Malignant pleural effusions are linked to carcinomas of the lung (36\%), breast (25\%), lymphoma (10\%), and ovary (5\%). Malignant effusions are either serous, serosanguinous or hemorragic. Malignancy comes second to pneumonia in etiology of exudative pleural effusions, and is responsible for transudative pleural effusion in $8 \%$ to $20 \%$ of all cytologically recognized malignant effusions. Mechanisms of effusion in malignancy include invasion of pleura, local and systemic tumor effects, hypoalbuminemia, cancer treatment complications, lymphatic obstruction, collapse, chemotherapy, radiation, pneumonia and congestive heart failure [25].

Pleurodesis is indicated in those patients with symptomatic relief after thoracentesis. Talc, bleomycin, nitrogen mustard, minocycline and 5-fluorouracil, silver nitrate and patient own blood have been utilized as sclerosing agents. The most effective are talc, doxycycline, minocycline, and bleomycin with rates of $72 \%$ to $90 \%$ success. Life expectancy should also be considered [26]. Alternatives for patients with poor life expectancy may comprise recurrent thoracentesis or placement of an indwelling catheter. Other therapeutic options for malignant pleural effusions include thoracotomy with decortication, generally reserved for patients unresponsive to traditional management $[27,28]$. 


\section{Open Access Journal of Pulmonary \& Respiratory Sciences}

\section{Pneumothorax}

Pleural metastases, infection, cancer treatment, other pulmonary comorbidities or iatrogenic causes can lead to pneumothorax in cancer patients. Most pneumothoraces are iatrogenic or due to secondary spontaneous reasons.. Iatrogenic procedures associated with pneumothorax incorporate percutaneous lung biopsy, transbronchial biopsy, insertion of central venous lines, pulmonary artery catheters and in reported cases nasogastric tube placement [29].

Chest radiograph is diagnostic as it shows a visceral pleural line with the absence of lung markings beyond the line. In some cases, expiratory films may be helpful. Transthoracic chest Sonography is absolutely sensitive and specific for diagnosis of pneumothorax [30].

\section{References}

1. Sorensen HT, Mellemkjaer L, Olsen JH, Baron JA (2000) Prognosis of cancers associated with venous thromboembolism. N Engl J Med 343(25): 1846-1850.

2. Blom JW, Doggen CJ, Osanto S, Rosendaal FR (2005) Malignancies, prothrombotic mutations, and the risk of venous thrombosis. JAMA 293(6): 715-722.

3. Tesselaar ME, Osanto S (2007) Risk of venous thromboembolism in lung cancer. CurrOpinPulm Med 13(5): 362-367.

4. Varki A (2007) Trousseau's syndrome: multiple definitions and multiple mechanisms. Blood 110(6): 1723-1729.

5. Blom JW, Osanto S, Rosendaal FR (2004) The risk of a venous thrombotic event in lung cancer patients: higher risk for adenocarcinoma than squamous cell carcinoma. J Thromb Haemost 2(10): 1760-1765.

6. Davidson MB, Thakkar S, Hix JK, Bhandarkar MD, Wong A, et al. (2004) Pathophysiology, clinical consequences, and treatment of tumor lysis syndrome. Am J Med 116(8): 546-554.

7. Coiffier B, Riouffol C (2007) Management of tumor lysis syndrome in adults". Expert Review of Anticancer Therapy 7(2): 233-239.

8. Alessandro V, Greco A, Clemente C, Sperandeo M, De Cata A, et al. (2010). Severe spontaneous acute tumor lysis syndrome and hypoglycemia in patient with germ cell tumor. Tumori 96(6): 1040-1043.
9. Cahill BC, Ingbar DH (1994) Massive hemoptysis. Assessment and management. Clin Chest Med 15(1): 147-167.

10. Escalante CP (1993) Causes and management of superior vena cava syndrome. Oncology 7(6): 61-68.

11. Vaitkus PT, Herrmann HC, Le Winter MM (1994) Treatment of malignant pericardial effusion. JAMA 272(1): 59-64.

12. Helms SR, Carlson MD (1989) Cardiovascular emergencies. Semin Oncol 16: 463-470.

13. Pierce ST (1993) Para endocrine syndromes. Curr Opin Oncol 5: 639-645.

14. Moses AM, Scheinman SJ (1991) Ectopic secretion of neurohypophyseal peptides in patients with malignancy. Endocrinol Metab Clin North Am 20(3): 489-506.

15. Stewart AF (2005) Clinical practice. Hypercalcemia associated with cancer. N Engl J Med 352(4): 373379.

16. Berenson JR, Hillner BE, Kyle RA, Anderson K, Lipton A, et al. (2002) American Society of Clinical Oncology clinical practice guidelines: the role of bisphosphonates in multiple myeloma. J ClinOncol 20(17): 3719-3736.

17. Kvale PA, Simoff M, Prakash UB (2003) American College of Chest Physicians. Lung cancer. Palliative care Chest 123(1): S284-311.

18. Quint DJ (2000) Indications for emergent MRI of the central nervous system. JAMA 283(7): 853-855.

19. Bilsky MH, Lis E, Raizer J, Lee H, Boland P (1999) The diagnosis and treatment of metastatic spinal tumor. Oncologist 4(6): 459-469.

20. Newton HB (1999) Neurologic complications of systemic cancer. Am Fam Physician 59(4): 878-886.

21. Hughes WT, Armstrong D, Bodey GP, Bow EJ, Brown $\mathrm{AE}$, et al. (2002) guidelines for the use of antimicrobial agents in neutropenic patients with cancer. Clin Infect Dis 34(6): 730-751.

22. Gertz MA, Fonseca R, Rajkumar SV (2000) Waldenström's macroglobulinemia. Oncologist 5(1): 63-67. 


\section{Open Access Journal of Pulmonary \& Respiratory Sciences}

23. Rhodes V, Manzullo E (1997) Oncologic emergencies. In: Pazdur R. Medical Oncology: A Comprehensive Review. $2^{\text {nd }}($ Edn.), Huntington, N.Y, PRR.

24. Lee P, Kupeli E, Metha AC (2002) Therapeutic bronchoscopy in lung cancer. Laser therapy, stents and photodynamic therapy. Clin Chest Med 23(1): 241-256.

25. Chernow B, Sahn SA (1977) Carcinomatous involvement of the pleura: an analysis of 96 patients. Am J Med 63(5): 695-702.

26. Johnston WW (1985) The malignant pleural effusion. A review of cytopathologic diagnoses of 584 specimens from 472 consecutive patients. Cancer 56(4): 905-909.
27. Viallat JR, Rey F, Astoul P, Boutin C (1996) Thoracoscopic talc poudragepleurodesis for malignant effusions. A review of 360 cases. Chest 110(6): 1387-1393.

28. Pien GW, Gant MJ, Washam CL, Sterman DH (2001) Use of an implantable pleural catheter for trapped lung syndrome in patients with malignant pleural effusion. Chest 119(6): 1641-1646.

29. Aronchick JM, Epstein DM, Gefter WB, Miller WT (1984) Pneumothorax as a complication of placement of a nasoenteric tube. JAMA 252(23): 3287-3288.

30. Tocino IM, Miller MH, Fairfax WR (1985) Distribution of pneumothorax in the supine and semirecumbent critically ill adult. AJR Am J Roentgenol 144(5): 901905. 